\title{
SELP - A System for Studying Strong Equivalence between Logic Programs
}

\author{
Yin Chen ${ }^{1,2}$, Fangzhen $\operatorname{Lin}^{3}$ and Lei $\mathrm{Li}^{2}$ \\ 1 Department of Computer Science, South China Normal University, China \\ ${ }^{2}$ Software Institute, Sun Yat-sen University, China \\ 3 Department of Computer Science, Hong Kong University of Science and Technology, Clear \\ Water Bay, Kowloon, Hong Kong
}

\begin{abstract}
This paper describes a system called SELP for studying strong equivalence in answer set logic programming. The basic function of the system is to check if two given ground disjunctive logic programs are equivalent, and if not, return a counter-example. This allows us to investigate some interesting properties of strong equivalence, such as a complete characterization for a rule to be strongly equivalent to another one, and checking whether a given set of rules is strongly equivalent to another, perhaps simpler set of rules.
\end{abstract}

\section{Introduction}

The notion of strongly equivalent logic programs was proposed by [5]. It has been found useful for tasks such as program simplification (e.g. [2]). In this paper, we describe a system called SELP that can help us answer questions regarding this notion, from simple ones such as "are P and Q strongly equivalent" to more involved ones such as "exactly what kind of rules are strongly equivalent to the empty set".

The core of the system is checking whether two disjunctive logic programs are strongly equivalent. This is based on [6], which provides a simple mapping from logic programs to propositional theories that reduces strong equivalence to entailment in classical propositional logic. Thus, the problem of strong equivalence checking can be translated into a satisfiability problem in propositional logic, and solved using SAT solvers like zChaff [10].

In addition, when two programs are not strongly equivalent, we may want to find some witnesses. For example, $P_{1}=\left\{\left(a_{1} \leftarrow a_{2}\right),\left(a_{1} \leftarrow\right.\right.$ not $\left.\left.a_{2}\right)\right\}$ and $P_{2}=\left\{a_{1} \leftarrow\right\}$ are not strongly equivalent, and $P=\left\{a_{2} \leftarrow a_{1}\right\}$ is a witness (counter-example): it is easy to see that $\left\{a_{1}, a_{2}\right\}$ is an answer set of $P_{2} \cup P$, but not of $P_{1} \cup P$. It is often hard to find a witness manually, but SELP can do this automatically: when two programs $P_{1}$ and $P_{2}$ are found not to be strongly equivalent, it will return a program $P$, such that $P_{1} \cup P$ and $P_{2} \cup P$ have different answer sets.

The most interesting part of SELP is that it allows us to study some properties of the notion of strong equivalence. In [7], we described some results on classes of strongly equivalent logic programs discovered using the system. In this paper, we shall show how the system can help us answer questions of the following form: Given a set $P$ of rules, is there another set of rules of certain property that is strongly equivalent to $P$ ? 
In [4], a system called LPEQ was developed that can check if two normal programs are strongly equivalent, and was implemented using the answer set logic programming system smodels. Besides being implemented using a different technique, our system can deal with normal as well as disjunctive logic programs. Furthermore, our system can construct a counter-example when two programs are not strongly equivalent.

The remainder of this paper is organized as follows. In the next section, we review some key concepts and notations in answer set logic programming. In section 3, we describe the core of the system, i.e. how to check if two programs are strongly equivalent. In section 4, we describe how to discover classes of strongly equivalent programs using SELP. In section 5, we show how to find all programs that are strongly equivalent to a given one. Finally, we conclude the paper in section 6 .

\section{Preliminaries}

Let $L$ be a propositional language, i.e. a set of atoms. In this paper we shall consider logic programs with rules of the following form:

$$
h_{1} ; \cdots ; h_{k} \leftarrow p_{1}, \cdots, p_{m}, \operatorname{not} p_{m+1}, \cdots, \operatorname{not} p_{n}
$$

where $h_{i}$ 's and $p_{i}$ 's are atoms in $L$. So a logic program here can have default negation (not), constraints (when $k=0$ ), and disjunctions in the head of its rules. In the following, if $r$ is a rule of the above form, we write $H d_{r}$ to denote the set $\left\{h_{1}, \ldots, h_{k}\right\}, P s_{r}$ the set $\left\{p_{1}, \ldots, p_{m}\right\}$, and $N g_{r}$ the set $\left\{p_{m+1}, \ldots, p_{n}\right\}$. Thus a rule $r$ can also be written as $H d_{r} \leftarrow P s_{r}$, not $N g_{r}$.

The semantics of these programs are given by answer sets as defined in [3]. As conventional in logic programming, we identify interpretations of $L$ with sets of atoms in $L$. Let $I$ be a set of atoms, and $P$ a logic program. We say that $I$ is closed under $P$ if for any rule $r$ in $P$, we have that $H d_{r} \cap I \neq \emptyset$ whenever $P s_{r} \subseteq I$ and $N g_{r} \cap I=\emptyset$. Now if $P$ is a program without negation, a set $I$ of atoms is an answer set of $P$ iff $I$ is a minimal set of atoms that is closed under $P$. Generally, $I$ is an answer set of $P$ iff $I$ is an answer set of $P^{I}$, where $P^{I}$, the reduct of $P$ on $I$, is obtained from $P$ as follows: for any rule of form (1), if there is an atom $p_{i}, m+1 \leq i \leq n$, such that $p_{i} \in I$, then delete this rule; otherwise, delete all the literals of the form not $q_{i}$ from this rule.

Two logic programs $P_{1}$ and $P_{2}$ in $L$ are said to be equivalent if they have the same answer sets, and strongly equivalent [5] (in the language $L$ ) if for any logic program $P$ in $L, P \cup P_{1}$ and $P \cup P_{2}$ are equivalent. For instance, $\{p \leftarrow q\}$ and $\{(p \leftarrow q),(q \leftarrow p)\}$ are equivalent but not strongly equivalent: they both have the unique answer set $\emptyset$, but when we add the rule $p \leftarrow$ to them, the first one will have the answer set $\{p\}$, while the latter $\{p, q\}$.

The notion of strongly equivalent logic programs is interesting for a variety of reasons. For instance, as Lifschitz et al. [5] noted, if two sets of rules are strongly equivalent, then one can be replaced by the other in any logic program regardless of the context. Thus knowing whether two sets of rules are strongly equivalent is a useful exercise that may have applications in program simplification.

In the following, for convenience, when we say a rule is strongly equivalent to the empty set, we mean the set that contains exactly this rule is strongly equivalent to the 
empty set. Similarly, when we say two rules are strongly equivalent, we mean that the two sets of rules, each consisting of exactly one of the rules, are strongly equivalent.

\section{Checking strong equivalence between two logic programs}

Lifschitz, Pearce, and Valverde [5] showed that checking for strong equivalence between two logic programs can be done in the logic of here-and-there, a three-valued non-classical logic somewhere between classical logic and intuitionistic logic. Turner [11] provided a model-theoretic characterization of strong equivalence in terms of pairs of sets of atoms. Lin [6] provided a mapping from logic programs to propositional theories and showed that two logic programs are strongly equivalent iff their corresponding theories in propositional logic are equivalent. This result will be the basis that we are using in this paper for checking if two logic programs are strongly equivalent, and we repeat it here.

Let $P_{1}$ and $P_{2}$ be two finite logic programs, and $L$ the set of atoms in them.

Theorem 1. [6] $P_{1}$ and $P_{2}$ are strongly equivalent iff in the propositional logic, the following two entailments hold:

$$
\begin{aligned}
& \left\{p \supset p^{\prime} \mid p \in L\right\} \cup \Delta\left(P_{1}\right) \models \Delta\left(P_{2}\right), \\
& \left\{p \supset p^{\prime} \mid p \in L\right\} \cup \Delta\left(P_{2}\right) \models \Delta\left(P_{1}\right) .
\end{aligned}
$$

where for each $p \in L, p^{\prime}$ is a new atom, and for each program $P, \Delta(P)=\{\Delta(r) \mid r \in$ $P\}$, where for each rule $r$ of the form $(1), \Delta(r)$ is the conjunction of the following two sentences:

$$
\begin{aligned}
& p_{1} \wedge \cdots \wedge p_{m} \wedge \neg p_{m+1}^{\prime} \wedge \cdots \wedge \neg p_{n}^{\prime} \supset h_{1} \vee \cdots \vee h_{k}, \\
& p_{1}^{\prime} \wedge \cdots \wedge p_{m}^{\prime} \wedge \neg p_{m+1}^{\prime} \wedge \cdots \wedge \neg p_{n}^{\prime} \supset h_{1}^{\prime} \vee \cdots \vee h_{k}^{\prime} .
\end{aligned}
$$

Notice that if $m=n=0$, then the left sides of the implications in (4) and (5) are considered to be true, and if $k=0$, then the right sides of the implications in (4) and (5) are considered to be false.

Theorem 1 makes it possible to check the strong equivalence between two logic programs using a SAT solver. For instance, to verify (2), it is sufficient to check that both of the following two formulas are satisfied for all $r \in P_{2}$ :

$\left\{p \supset p^{\prime} \mid p \in L\right\} \cup \Delta\left(P_{1}\right) \cup\left\{p \mid p \in P s_{r}\right\} \cup\left\{\neg p^{\prime} \mid p \in N g_{r}\right\} \cup\left\{\neg p \mid p \in H d_{r}\right\}$, $\left\{p \supset p^{\prime} \mid p \in L\right\} \cup \Delta\left(P_{1}\right) \cup\left\{p^{\prime} \mid p \in P s_{r}\right\} \cup\left\{\neg p^{\prime} \mid p \in N g_{r} \cup H d_{r}\right\}$.

Algorithm 1 makes precise this idea, and was implemented using the SAT solver zChaff [10].

If $P_{1}$ and $P_{2}$ are not strongly equivalent, then zChaff will return an assignment that is a counter-example to either (2) or (3), and from this assignment, we can construct a program $P$ such that $P \cup P_{1}$ and $P \cup P_{2}$ are not equivalent, i.e. $P$ is a witness of the fact that $P_{1}$ and $P_{2}$ are not strongly equivalent. 
1: procedure SELP(LP $\left.P_{1}, \operatorname{LP} P_{2}\right)$

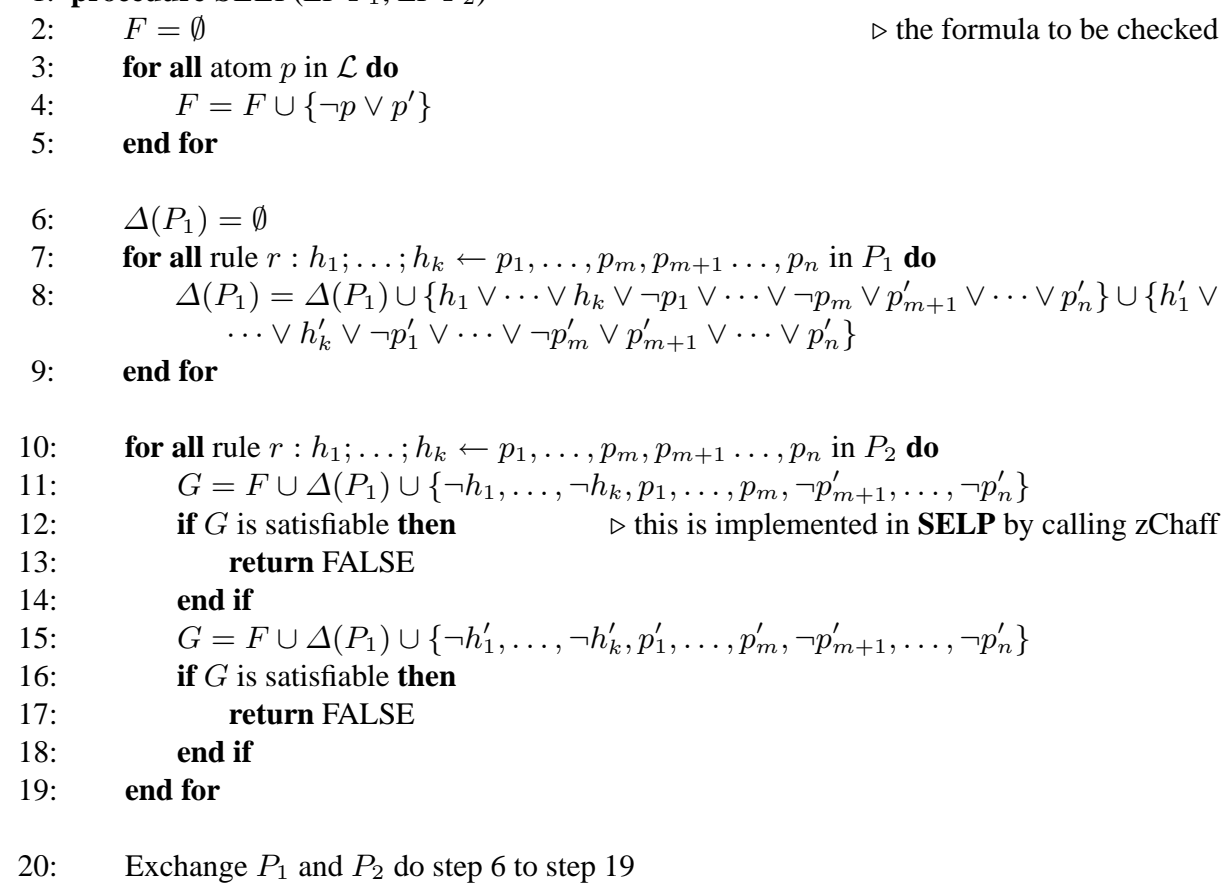


Theorem 2. Let $P_{1}$ and $P_{2}$ be two programs, $M$ a model of $\left\{p \supset p^{\prime} \mid p \in L\right\} \cup \Delta\left(P_{1}\right)$, and not $\Delta\left(P_{2}\right)$. Let $M_{L}$ and $M_{L^{\prime}}$ be the two sets of atoms defined as follows:

$$
\begin{aligned}
M_{L} & =\{p \mid p \in L \text { and } M \models p\}, \\
M_{L^{\prime}} & =\left\{p \mid p \in L \text { and } M \models p^{\prime}\right\} .
\end{aligned}
$$

Then we have

(1) If $M_{L^{\prime}}$ is not closed under $P_{2}$, then $P_{1} \cup P$ and $P_{2} \cup P$ is not equivalent, where $P=\left\{p \leftarrow \mid p \in M_{L^{\prime}}\right\}$.

(2) If $M_{L^{\prime}}$ is closed under $P_{2}$, then $P_{1} \cup P$ and $P_{2} \cup P$ is not equivalent, where $P=$ $\left\{p \leftarrow \mid p \in M_{L}\right\} \cup\left\{p \leftarrow q \mid p, q \in M_{L^{\prime}} \backslash M_{L}, p \neq q\right\}$.

Proof. Follows from the proofs of Theorem 1 in [5] and Theorem 1 in [6].

For example, given $P_{1}=\{(a \leftarrow \operatorname{not} b),(b \leftarrow \operatorname{not} a)\}$ and $P_{2}=\{a ; b \leftarrow\}$, the answer of SELP will return the counter-example $P=\{(a \leftarrow b),(b \leftarrow a)\}$. However, SELP will confirm that $P_{1} \cup\{\leftarrow a, b\}$ and $P_{2} \cup\{\leftarrow a, b\}$ are strongly equivalent. As another example, consider $P_{3}=\{(a \leftarrow b, c),(a \leftarrow b$, not $c)\}$ and $P_{4}=\{a \leftarrow b\}$. They are not strongly equivalent, and $\{(a \leftarrow c),(c \leftarrow a),(b \leftarrow)\}$ is the counter-example returned by SELP.

While the basic function of our system SELP is to check whether two given logic programs are strongly equivalent, and if not provides a witness, we do not envision its use this way. Rather, we consider it a tool to systematically study the notion of strong equivalence. In the following, we discuss its use in discovering classes of strongly equivalent logic programs [7], and in searching for simpler sets of rules that are strongly equivalent to a given one.

\section{Discovering general theorems}

As we have mentioned, one possible use of strongly equivalent logic program is in program simplification. For instance, if a rule is strongly equivalent to an empty set, then we can delete this rule in any program without changing the answer sets of the program. It is well-known that a rule $r$ is strongly equivalent to empty set if $H d_{r} \cap N g_{r} \neq \emptyset$. But is this the only case, i.e. is this both a sufficient and necessary condition for a rule to be strongly equivalent to the empty set?

In [7], we described a methodology and proved some general theorems for discovering theorems like this. More precisely, we were interested in the following so-called $k-m-n$ theorem-discovery problem: Find some computationally effective conditions under which a set $\left\{r_{1}, \ldots, r_{k}, u_{1}, \ldots, u_{m}\right\}$ of $k+m$ rules is strongly equivalent to a set $\left\{r_{1}, \ldots, r_{k}, v_{1}, \ldots, v_{n}\right\}$ of $k+n$ rules. Notice that these two sets share $k$ rules. This is to capture the so-called conditional strong equivalence. For instance, as we mentioned above, if $r$ is strongly equivalent to $\emptyset$, then we can delete $r$ from any logic program. However, if we want to delete $r$ in a logic program only under the condition that another rule $r^{\prime}$ is in the program, then what we need is to check that $\left\{r^{\prime}, r\right\}$ and $\left\{r^{\prime}\right\}$ are strongly equivalent. 
We will not go into details of how we addressed the $k-m-n$ problems (see [7]). The basic idea is to first find a condition that captures the strong equivalence between $\left\{r_{1}, \ldots, r_{k}, u_{1}, \ldots, u_{m}\right\}$ and $\left\{r_{1}, \ldots, r_{k}, v_{1}, \ldots, v_{n}\right\}$ when all the rules are from a small language, say with only three atoms. To do this, we generate all possible triples of the form $\left(S_{1}, S_{2}, S_{3}\right)$, where $S_{1}, S_{2}$ and $S_{3}$ are sets of $k$ number, $m$ number and $n$ number, respectively, of rules, and run SELP to check whether $S_{1} \cup S_{2}$ is strongly equivalent to $S_{1} \cup S_{3}$. The following are some of the experimental results that we obtained using SELP.

Lemma 1. If $r$ mentions at most three distinct atoms, then $r$ is s.e. to $\emptyset$ iff $\left(H d_{r} \cup\right.$ $\left.N g_{r}\right) \cap P s_{r} \neq \emptyset$.

Lemma 2. For any two rules $r_{1}$ and $r_{2}$ that mentions at most four atoms, $\left\{r_{1}, r_{2}\right\}$ and $\left\{r_{1}\right\}$ are strongly equivalent iff one of the following two conditions is true:

1. $\left\{r_{2}\right\}$ is strongly equivalent to $\emptyset$.

2. $P s_{r_{1}} \subseteq P s_{r_{2}}, N g_{r_{1}} \subseteq N g_{r_{2}}, H d_{r_{1}} \subseteq H d_{r_{2}} \cup N g_{r_{2}}$.

Lemma 3. For any three rules $r_{1}, r_{2}$ and $r_{3}$ that mentions at most six atoms, $\left\{r_{1}, r_{2}, r_{3}\right\}$ and $\left\{r_{1}, r_{2}\right\}$ are strongly equivalent iff one of the following four conditions is true:

1. $\left\{r_{3}\right\}$ is strongly equivalent to $\emptyset$.

2. $P s_{r_{1}} \subseteq P s_{r_{3}}, N g_{r_{1}} \subseteq N g_{r_{3}}, H d_{r_{1}} \subseteq H d_{r_{3}} \cup N g_{r_{3}}$.

3. $P s_{r_{2}} \subseteq P s_{r_{3}}, N g_{r_{2}} \subseteq N g_{r_{3}}, H d_{r_{2}} \subseteq \mathrm{Hd}_{r_{3}} \cup N g_{r_{3}}$.

4. there is an atom $p$ such that:

$$
\begin{aligned}
& 4.1 p \in\left(P s_{r_{1}} \cup P s_{r_{2}}\right) \cap\left(H d_{r_{1}} \cup H d_{r_{2}} \cup N g_{r_{1}} \cup N g_{r_{2}}\right) \\
& 4.2 H d_{r_{i}} \backslash\{p\} \subseteq H d_{r_{3}} \cup N g_{r_{3}} \text { and } P s_{r_{i}} \backslash\{p\} \subseteq P s_{r_{3}} \text { and } N g_{r_{i}} \backslash\{p\} \subseteq N g_{r_{3}} \text {, } \\
& \text { where } i=1,2 \\
& \text { 4.3 If } p \in P s_{r_{1}} \cap N g_{r_{2}} \text {, then } H d_{r_{1}} \cap H d_{r_{3}}=\emptyset \\
& \text { 4.4 If } p \in P s_{r_{2}} \cap N g_{r_{1}} \text {, then } H d_{r_{2}} \cap H d_{r_{3}}=\emptyset
\end{aligned}
$$

Some general theorems are proved in [7] that help us verifying that Lemmas 1-3 in fact hold in the general case.

Theorem 3. Lemma 1-3 hold in the general case, without any restriction on the number of atoms.

An important consequence of this theorem is the following theorem that solves the 0-1-1 problem:

Theorem 4 (0-1-1). For any two rules $r_{1}$ and $r_{2},\left\{r_{1}\right\}$ and $\left\{r_{2}\right\}$ are strongly equivalent iff one of the following two conditions is true:

1. $\left\{r_{1}\right\}$ and $\left\{r_{2}\right\}$ are both strongly equivalent to $\emptyset$.

2. $P s_{r_{1}}=P s_{r_{2}}, N g_{r_{1}}=N g_{r_{2}}$, and $H d_{r_{1}} \cup N g_{r_{1}}=H d_{r_{2}} \cup N g_{r_{2}}$.

Proof. By Theorem 1, it is easy to see that $\left\{r_{1}\right\}$ and $\left\{r_{2}\right\}$ are strongly equivalent iff $\left\{r_{1}, r_{2}\right\}$ and $\left\{r_{1}\right\}$ are strongly equivalent and $\left\{r_{1}, r_{2}\right\}$ and $\left\{r_{2}\right\}$ are strongly equivalent. 
Notice that for a language with six atoms, there are in principle $\left(2^{6}\right)^{3}-1=262,143$ possible rules. So for Lemma 3, which is about the 2-1-0 problem, there would be $262,143^{3}$ cases to check, which would be impossible to do using currently available computers. Fortunately, we can cut the numbers down significantly. First, by Theorems 3 and 4, we only need to consider rules where each atom occurs at most once: for any rule $r$, if there is an atom $p$ that occurs more than once in $r$, then one of the following two cases applies:

1. $p \in P s_{r} \cap\left(N g_{r} \cup H d_{r}\right)$, in this case, $r$ is strongly equivalent to the empty set;

2. $p \in N g_{r} \cap H d_{r}$, in this case, $r$ is strongly equivalent to $\left(H d_{r} \backslash N g_{r}\right) \leftarrow P s_{r}$, not $N g_{r}$.

In the following, we call rules in which each atom occurs at most once non-redundant rules. Second, we do not have to consider isomorphic rules: if there is a one-to-one onto function from $L$ to $L$ that maps $\left\{r_{1}, \ldots\right\}$ to $\left\{r_{1}^{\prime}, \ldots\right\}$, then these two sets of rules are essentially the same except the names of atoms in them.

In general, we have the following theorem, which says that for checking a condition about the $k-m-n$ problem in a language with $N$ atoms, it is sufficient to consider $\left(\begin{array}{c}4^{k+m+n}+N-1 \\ N\end{array}\right)$ cases. For instance, for Lemma 3, there are $\left(\begin{array}{c}69 \\ 6\end{array}\right)=119,877,472$ cases to check, which took about 10 hours on a Solaris server consisting of 8 Sun UltraSPARC III 900Mhz CPUs with 8GB RAM.

Theorem 5. Let $L$ be a language with $N$ atoms $a_{1}, \ldots, a_{N}$. For any $n_{1}, n_{2}, n_{3} \geq 0$, we can generate a set $S$ of less than $\left({ }^{4}+{ }_{N}-1\right)$ triples $\left(R_{1}, R_{2}, R_{3}\right)$, where $M=$ $n_{1}+n_{2}+n_{3}$ and for each $1 \leq i \leq 3, R_{i}$ is a set of no more than $n_{i}$ number of rules in $L$, such that for any sets $W_{1}, W_{2}, W_{3}$ of $n_{1}, n_{2}, n_{3}$ rules, respectively, in $L$, there is a triple $\left(R_{1}, R_{2}, R_{3}\right)$ in $S$ and an automorphism $f$ of $L$ such that $R_{1} \cup R_{2}$ and $f\left(W_{1}\right) \cup f\left(W_{2}\right)$ are strongly equivalent, and $R_{1} \cup R_{3}$ and $f\left(W_{1}\right) \cup f\left(W_{3}\right)$ are strongly equivalent, where for any set $W$ of rules, $f(W)$ is the result of replacing each atom a in every rule of $W$ by $f(a)$.

The proof of this theorem is omitted, and we give some intuitive explanations here. The problem is to generate $M$ rules using $N$ atoms. As mentioned above, we consider only non-redundant rules. For example, when $M=1$, the problem is to generate one rule using $N$ atoms. There are 4 cases of the occurrence of an atom in a non-redundant rule:

(i) in head of the rule,

(ii) in the body of the rule positively,

(iii) in the body of the rule negatively,

(vi) none of above.

Let $r$ and $r^{\prime}$ be two rules, and, for both of them, there are $x_{0}$ atoms of case (i), $x_{1}$ atoms of case (ii), $x_{2}$ atoms of case (iii), and $x_{3}$ atoms of case (vi), where $x_{0}+x_{1}+x_{2}+x_{3}=$ $N$. We can easily define an automorphism of $L$ and map $r$ and $r^{\prime}$ to the same rule. So $r$ and $r^{\prime}$ are isomorphic rules. Thus, for $M=1$, the triples in $S$ is less than the nonnegative integer answers of equation $x_{0}+x_{1}+x_{2}+x_{3}=N$.

For $M=2$, there are $4^{2}=16$ cases of the occurrence of an atom in two rules $r_{1}, r_{2}$ : 
(i) in head of $r_{1}$ and in the head of $r_{2}$,

(ii) in head of $r_{1}$ and in the body of $r_{2}$ positively,

(iii) in head of $r_{1}$ and in the body of $r_{2}$ negatively,

(vi) in head of $r_{1}$ and not in $r_{2}$,

(vi) in the body of $r_{1}$ positively and in head of $r_{2}$,

... $\ldots$

(xvi) not in $r_{1}$ and not in $r_{2}$

So, for $M=2$, the triples in $S$ is less than the non-negative integer answers of equation $x_{0}+x_{1}+x_{2}+\cdots+x_{15}=N$. In general, there are $4^{M}$ cases of the occurrence of an atom in $M$ rules, and the triples in $S$ is less than the non-negative integer answers of equation $x_{0}+x_{1}+x_{2}+\cdots+x_{4^{M}-1}=N$. Notice that there are exactly $\left(\begin{array}{c}4^{M}+N-1 \\ N\end{array}\right)$ non-negative integer answers of $x_{0}+x_{1}+x_{2}+\cdots+x_{4^{M}-1}=N$.

\section{Finding strongly equivalent logic programs}

Our second application of SELP is about finding out whether there is another, preferably simpler logic program that is strongly equivalent to a given one. For instance, we have seen that the self-loops (loops of length one) like $p \leftarrow p, q$ are strongly equivalent to $\emptyset$. A natural follow-up question is then: what about loops of length two, like $\{(a \leftarrow b),(b \leftarrow a)\}$ ?

Given a program $P$ in the language $L$, an obvious way to look for another program in $L$ that is strongly equivalent to $P$ would be to generate all possible programs in $L$, and call SELP on them one by one. This is clearly infeasible even for a program with only three or four atoms. Fortunately, there is a much better way of doing it. Instead of considering all possible sets of rules, we can first find all possible rules that are redundant in the presence of $P$, i.e. all rules $r$ such that $P \cup\{r\}$ is strongly equivalent to $P$, and consider sets of these rules only, as the following theorem says.

Theorem 6. Let $P$ be a logic program in $L$, and $S$ the set of rules defined as follows:

$$
S=\{r \mid r \text { is in } L, \text { and } P \cup\{r\} \text { and } P \text { are strongly equivalent }\} .
$$

For any program $Q$ in $L$, if $P$ and $Q$ are strongly equivalent, then $Q \subseteq S$.

Proof. Follows from Theorem 1.

Notice that the set $S$ in the theorem includes "trivial" rules like $p \leftarrow p$. As we mentioned in Section 4, by Theorem 3 and Theorem 4, we need to consider only rules where each atom occurs at most once, i.e. non-redundant rules.

Corollary 1. Let $P$ be a logic program in $L$, and $S_{P}$ the set of rules defined as follows: $S_{P}=\{r \mid r$ is a non-redundant rule in $L$, and $P \cup\{r\}$ and $P$ are strongly equivalent $\}$.

For any program $Q$ in $L$, if $P$ and $Q$ are strongly equivalent, then $Q^{\prime} \subseteq S_{P}$, where $Q^{\prime}$ is obtained from $Q$ by deletion rules that are strongly equivalent to $\emptyset$, and replace each remaining rule $r$ by $H d_{r} \backslash N g_{r} \leftarrow P s_{r}$, not $N g_{r}$. 
Using this corollary, our system SELP finds all programs that are strongly equivalent to a given logic program in two steps:

- generate all possible non-redundant rule $r$, and check if $P$ is strongly equivalent to $P \cup\{r\}$, thus computing the set $S_{P}$,

- for each subset of $S_{P}$, check if it is strongly equivalent to $P$.

For our example loop with length two, $P_{1}=\{(a \leftarrow b),(b \leftarrow a)\}, S_{P_{1}}$ consists of the following rules:

$$
a \leftarrow b \quad b \leftarrow a \quad \leftarrow a, \text { not } b \leftarrow b, \operatorname{not} a
$$

As it turned out, there is no subset of $S_{P_{1}}$ that is strongly equivalent to $P_{1}$ yet does not contain $P_{1}$, i.e. $P_{1}$ cannot be simplified using strong equivalence.

As another example, consider

$$
P_{2}=\left\{\left(a_{1} \leftarrow \operatorname{not} a_{2}\right),\left(a_{2} \leftarrow \operatorname{not} a_{3}\right),\left(a_{3} \leftarrow \operatorname{not} a_{1}\right)\right\}
$$

This is a program with an odd cycle $[8,9]$, i.e. there is a simple cycle in the dependency graph of the program that has an odd number of negative edges. Odd cycles in a logic program act as constraints [9]. For instance, we have already seen that $a \leftarrow \operatorname{not} a$, the odd cycle with length one, is strongly equivalent to the constraint $\leftarrow$ not $a$. The hope is that odd cycles of greater lengths, like $P_{2}$, can be similarly reduced to a set of constraints. Unfortunately, like $P_{1}, P_{2}$ cannot be simplified using strong equivalence either. This means that how rules in $P_{2}$ act as a constraint depends on other rules, thus cannot be determined locally.

The details of our experiment with $P_{2}$ is as follows. SELP returned $S_{P_{2}}$ as the set of following rules:

\begin{tabular}{lll}
\hline$\leftarrow$ not $a_{1}$, not $a_{2}$ & $\leftarrow a_{3}$, not $a_{1}$, not $a_{2}$ & $\leftarrow$ not $a_{1}$, not $a_{3}$ \\
$\leftarrow a_{2}$, not $a_{1}$, not $a_{3}$ & $\leftarrow$ not $a_{2}$, not $a_{3}$ & $\leftarrow a_{1}$, not $a_{2}$, not $a_{3}$ \\
$\leftarrow$ not $a_{1}$, not $a_{2}$, not $a_{3}$ & $a_{1} \leftarrow$ not $a_{2}$ & $a_{1} \leftarrow a_{3}$, not $a_{2}$ \\
$a_{1} \leftarrow$ not $a_{2}$, not $a_{3}$ & $a_{2} \leftarrow$ not $a_{3}$ & $a_{2} \leftarrow a_{1}$, not $a_{3}$ \\
$a_{2} \leftarrow$ not $a_{1}$, not $a_{3}$ & $a_{3} \leftarrow$ not $a_{1}$ & $a_{3} \leftarrow a_{2}$, not $a_{1}$ \\
$a_{3} \leftarrow$ not $a_{1}$, not $a_{2}$ & $a_{1} ; a_{2} \leftarrow$ not $a_{3}$ & $a_{1} ; a_{3} \leftarrow$ not $a_{2}$ \\
$a_{2} ; a_{3} \leftarrow$ not $a_{1}$ & & \\
\hline
\end{tabular}

The following are programs that consist of rules from $S_{P_{2}}$, do not contain $P_{2}$ as a subset, and are strongly equivalent to $P_{2}$. 


\begin{tabular}{lll}
\hline$a_{1} \leftarrow a_{3}$, not $a_{2}$ & $a_{2} \leftarrow a_{1}$, not $a_{3}$ & $a_{1} \leftarrow a_{3}$, not $a_{2}$ \\
$a_{2} \leftarrow a_{1}$, not $a_{3}$ & $a_{3} \leftarrow a_{2}$, not $a_{1}$ & $a_{3} \leftarrow a_{2}$, not $a_{1}$ \\
$a_{3} \leftarrow a_{2}$, not $a_{1}$ & $a_{1} ; a_{2} \leftarrow$ not $a_{3}$ & $a_{1} ; a_{3} \leftarrow$ not $a_{2}$ \\
$a_{1} ; a_{2} \leftarrow$ not $a_{3}$ & $a_{2} ; a_{3} \leftarrow$ not $a_{1}$ & $a_{2} ; a_{3} \leftarrow$ not $a_{1}$ \\
$a_{1} ; a_{3} \leftarrow$ not $a_{2}$ & $a_{1} \leftarrow$ not $a_{2}$ & $a_{2} \leftarrow$ not $a_{3}$ \\
$a_{2} ; a_{3} \leftarrow$ not $a_{1}$ & & \\
$a_{3} \leftarrow a_{2}$, not $a_{1}$ & $a_{1} \leftarrow a_{3}$, not $a_{2}$ & $a_{2} \leftarrow a_{1}$, not $a_{3}$ \\
$a_{2} ; a_{3} \leftarrow$ not $a_{1}$ & $a_{2} \leftarrow a_{1}$, not $a_{3}$ & $a_{1} ; a_{2} \leftarrow$ not $a_{3}$ \\
$a_{1} \leftarrow$ not $a_{2}$ & $a_{1} ; a_{2} \leftarrow$ not $a_{3}$ & $a_{1} \leftarrow$ not $a_{2}$ \\
$a_{2} \leftarrow$ not $a_{3}$ & $a_{1} ; a_{3} \leftarrow$ not $a_{2}$ & $a_{3} \leftarrow$ not $a_{1}$ \\
& $a_{3} \leftarrow$ not $a_{1}$ & \\
$a_{1} \leftarrow a_{3}$, not $a_{2}$ & & \\
$a_{1} ; a_{3} \leftarrow$ not $a_{2}$ & & \\
$a_{2} \leftarrow$ not $a_{3}$ & & \\
$a_{3} \leftarrow$ not $a_{1}$ & & \\
\hline
\end{tabular}

As one can see, none of them contain constraints. Actually, they can all be explained by the theorems in [7]. For example, consider $Q=\left\{\left(a_{1} \leftarrow\right.\right.$ not $\left.a_{2}\right),\left(a_{2} \leftarrow\right.$ $a_{1}$, not $\left.a_{3}\right),\left(a_{1} ; a_{2} \leftarrow\right.$ not $\left.a_{3}\right),\left(a_{3} \leftarrow\right.$ not $\left.\left.a_{1}\right)\right\}$. $Q$ is strongly equivalent to $P$ because the first two rules are already in $P$, and the set of the last two rules is strongly equivalent to the last rule in $P$.

\section{Concluding remarks and future work}

We develop a tool SELP for studying strong equivalence between logic programs. It can check if two programs are strongly equivalent, as well as, it is helpful for us to find some general theorems on strong equivalence. We will try to find some more theorems by SELP. It is also an interesting works to find some general theorems on uniform equivalence [1], and to find the difference between two kinds of equivalence.

\section{References}

1. Thomas Eiter and Michael Fink. Uniform equivalence of logic programs under the stable model semantics. In Catuscia Palamidessi, editor, ICLP, volume 2916 of Lecture Notes in Computer Science, pages 224-238. Springer, 2003.

2. Thomas Eiter, Michael Fink, Hans Tompits, and Stefan Woltran. Simplifying logic programs under uniform and strong equivalence. In Vladimir Lifschitz and Ilkka Niemelä, editors, LPNMR, volume 2923 of Lecture Notes in Computer Science, pages 87-99. Springer, 2004.

3. Michael Gelfond and Vladimir Lifschitz. Classical negation in logic programs and disjunctive databases. New Generation Computing, 9:365-385, 1991.

4. Tomi Janhunen and Emilia Oikarinen. Lpeq and dlpeq - translators for automated equivalence testing of logic programs. In Vladimir Lifschitz and Ilkka Niemelä, editors, LPNMR, volume 2923 of Lecture Notes in Computer Science, pages 336-340. Springer, 2004. 
5. V. Lifschitz, D. Pearce, and A. Valverde. Strongly equivalent logic programs. ACM Transactions on Computational Logic, 2(4):526-541, 2001.

6. Fangzhen Lin. Reducing strong equivalence of logic programs to entailment in classical propositional logic. In Proceedings of the Eighth International Conference on Principles of Knowledge Representation and Reasoning (KR2002), pages 170-176, 2002.

7. Fangzhen Lin and Yin Chen. Discovering classes of strongly equivalent logic programs. In Proceedings of the Nineteenth International Joint Conference on Artificial Intelligence (IJCAI-05), 2005. To appear.

8. Fangzhen Lin and Jia-Huai You. Abduction in logic programming: A new definition and an abductive procedure based on rewriting. Artificial Intelligence, 140(1/2):175-205, 2002.

9. Fangzhen Lin and Xishun Zhao. On odd and even cycles in normal logic programs. In Proceedings of the 19th National Conference on Artificial Intelligence (AAAI-2004), AAAI Press, Menlo Park, CA., pages 80-85, 2004.

10. Matthew W. Moskewicz, Conor F. Madigan, Ying Zhao, Lintao Zhang, and Sharad Malik. Chaff: Engineering an efficient sat solver. In DAC, pages 530-535. ACM, 2001.

11. Hudson Turner. Strong equivalence for logic programs and default theories (made easy). In Proceedings of LPNMR'2001, pages 81-92, 2001. 